\title{
Conhecimento de mulheres sobre alguns aspectos do papiloma vírus humano
}

\section{The knowledge of women on some aspects of human papillomavirus}

\author{
Ana Teresa Mancini Pimenta ${ }^{1}$, Patricia Pereira dos Santos Melli², Geraldo Duarte ${ }^{3}$, Silvana Maria Quintana ${ }^{3}$
}

\begin{abstract}
RESUMO
Modelo do estudo: transversal. Objetivo do estudo: verificar o conhecimento de mulheres atendidas em dois ambulatórios de Ginecologia e Obstetrícia sobre o Papiloma Vírus Humano (HPV). Metodologia: foram utilizados dados secundários de um banco de dados eletrônico em que as informações foram coletadas por meio de um questionário no período de 2007 a 2012. Resultados: foram incluídas 1.470 mulheres com média de idade de 30,7 anos. Dentre as mulheres, $51,0 \%$ já haviam ouvido falar de HPV, porém, apenas $41,6 \%$ sabiam da transmissão sexual e 33,3\% da relação do patógeno com o câncer de colo uterino. Conclusão: os dados permitiram concluir que o conhecimento sobre HPV é deficiente, necessitando desenvolvimento de ações educativas voltadas para a prevenção da infecção pelo HPV e seu agravamento.
\end{abstract}

Palavras-chave: Infecções por Papillomavirus. Conhecimento. Saúde da mulher.

\section{Introdução}

A infecção pelo Papilomavírus Humano (HPV) é considerada uma doença sexualmente transmissível (DST) altamente prevalente na população sexualmente ativa, estimando-se que $75 \%$ deste segmento populacional entrem em contato com um ou mais tipos de HPV durante a vida. ${ }^{1}$ A maioria das mulheres é infectada nos primeiros anos de atividade sexual, com taxas de prevalência mais elevadas em jovens e decrescentes com o aumento da idade. .,3,4 $^{2,}$ De acordo com Myers et al. $(2000)^{5}, 80 \%$ de todas as mulheres sexu- almente ativas terão sido expostas ao HPV quando estiverem com 50 anos. Outros estudos identificaram um segundo pico de prevalência em mulheres no período da perimenopausa que poderia decorrer de novas infecções adquiridas devido a mudanças no comportamento sexual ou de reativação de infecções latentes devido à senescência imune., ${ }^{4,6}$

As mulheres são mais vulneráveis às DST por causa de características biológicas como mucosa genital menos espessa e com maior superfície de contato. Outros fatores são as relações desiguais de gênero associadas à opressão por parte de seus parcei-
1. Aluna de Doutorado, Departamento de Ginecologia e Obstetrícia da Faculdade de Medicina de Ribeirão Preto da Universidade de São Paulo (DGO-FMRP-USP).

2. Médica assistente do DGO-FMRP-USP.

3. Professor titular do DGO-FMRP-USP.
Correspondência:

Departamento de Ginecologia e Obstetrícia da Faculdade de Medicina de Ribeirão Preto da Universidade de São Paulo (USP). Av. dos Bandeirantes, 3900, 8o andar. CEP 14049-900, Ribeirão Preto-SP, Brasil. E-mail: anateresa@usp.br

Artigo recebido em 26/04/2013 Aprovado para publicação em 04/02/2014 
ros e dependência econômica da mulher que limita o acesso às informações adequadas e atualizadas. ${ }^{7} \mathrm{Com}$ isso, é possível observar o desconhecimento das mulheres sobre $\mathrm{oHPV}^{8} \mathrm{e}$ as doenças sexualmente transmissíveis e modo de prevenção. ${ }^{9}$

O objetivo deste estudo foi verificar o conhecimento das mulheres atendidas em dois ambulatórios de Ginecologia e Obstetrícia do Hospital das Clínicas da Faculdade de Medicina de Ribeirão Preto da Universidade de São Paulo sobre HPV.

\section{Metodologia}

Foi realizado um estudo transversal utilizandose dados secundários de um banco de dados eletrônico. As informações contidas no banco de dados foram coletadas por meio de um questionário aplicado a todas as mulheres atendidas no Ambulatório de Moléstias Infectocontagiosas em Ginecologia e Obstetrícia (AMIGO) e no Ambulatório de Moléstias Infectocontagiosas em Ginecologia (AMIG) do Hospital das Clínicas da Faculdade de Medicina de Ribeirão Preto da Universidade de São Paulo (HC-FMRP-USP) no primeiro atendimento. Estas mulheres foram referenciadas a estes ambulatórios devido a apresentarem infecção pelo HIV e/ou alguma alteração ginecológica devido a agente infectocontagioso. O questionário abordou dados pessoais, antecedentes pessoais, ginecológicos e obstétricos, incluindo três questões sobre a infecção pelo HPV: se a paciente já havia ouvido falar sobre este vírus e qual foi a fonte desta informação, se sabia que a infecção é uma DST, isto é, se sabia que o HPV poderia ser adquirido durante a relação sexual e, finalmente, se conhecia a associação entre a infecção pelo HPV e o câncer de colo uterino. Foram incluídas todas as mulheres, gestantes e não gestantes que apresentassem os campos de resposta sobre conhecimento do agente infeccioso HPV, relação com o câncer de colo e a transmissão sexual do vírus preenchidos no banco de dados eletrônico, no período de janeiro de 2007 a agosto de 2012.

O estudo foi aprovado pelo Comitê de Ética em Pesquisa do Hospital das Clínicas da Faculdade de Medicina de Ribeirão Preto da Universidade de São Paulo sob número do parecer 56419/2012.

\section{Resultados}

Neste estudo a idade das mulheres variou de 11 a 95 anos, com média de 30,7 anos $(\mathrm{dp}=11,3)$. A tabela 1 mostra a distribuição das 1.470 mulheres incluídas no estudo de acordo com características sociodemográficas. Observou-se que a maioria delas era jovem em idade reprodutiva (90\%), com predomínio da faixa etária entre 21 a 30 anos $(37,2 \%)$, brancas $(61,5 \%)$, escolaridade entre 5 e 8 anos $(44,7 \%)$, casadas ou amasiadas $(60,5 \%)$.

A sexarca para $53,2 \%$ das mulheres aconteceu antes dos 16 anos de idade, para 39,7\% entre 16 e 20 anos e para 7,1\% após os 20 anos. Sete mulheres nunca haviam tido parceiros sexuais; $71,4 \%$ tiveram entre 1 e 5 parceiros; $14,7 \%$ entre 5 e $10 ; 5,7 \%$ entre 10 e 20 e 7,7\% número incontável de parceiros sexuais. A prostituição esteve presente na vida de 69 mulheres $(4,7 \%)$. Quanto à gestação, 15,6\% eram nuligestas, 26,1\% primigestas, $20,2 \%$ secundigestas, $16,1 \%$ tercigestas e $22,0 \%$ tinham quatro ou mais gestações.

Tabela 1. Distribuição das 1470 mulheres atendidas nos Ambulatórios de Moléstias Infecciosas em Ginecologia e Obstetrícia do Hospital das Clínicas da Faculdade de Medicina de Ribeirão Preto da Universidade de São Paulo, 20072012.

\begin{tabular}{ccc}
\hline Características & $\mathbf{n}^{\mathbf{0}}$ & $\%$ \\
\hline $\begin{array}{l}\text { Faixa etária (anos) } \\
11-20\end{array}$ & 286 & \\
$21-30$ & 547 & 37,5 \\
$31-45$ & 490 & 33,3 \\
$>45$ & 147 & 10
\end{tabular}

\section{Cor da pele}

$\begin{array}{llr}\text { branca } & 905 & 61,5 \\ \text { parda } & 342 & 23,3 \\ \text { negra } & 217 & 14,8 \\ \text { amarela } & 6 & 0,4\end{array}$

Escolaridade (em anos)

$\begin{array}{llr}\text { analfabeta } & 31 & 2,1 \\ 0-4 & 184 & 12,5 \\ 5-8 & 657 & 44,7 \\ 9-11 & 460 & 31,3 \\ >12 & 138 & 9,4\end{array}$

Estado marital

casada/ amasiada $\quad 890 \quad 60,5$ $\begin{array}{lll}\text { solteira } & 432 & 29,4\end{array}$ $\begin{array}{lll}\text { viúva } & 63 & 4,3\end{array}$

$\begin{array}{lll}\text { desquitada } & 85 & 5,8\end{array}$


No grupo avaliado, 656 mulheres $(44,6 \%)$ referiram ter recebido o diagnóstico de pelo menos uma doença sexualmente transmissível previamente. A DST mais prevalente foi a infecção pelo $\mathrm{HIV} \mathrm{em} 36,1 \%$ das pacientes, seguida da infecção pelo HPV em $21,2 \%$ (tabela 2).

Tabela 2. Frequência de doenças sexualmente transmissíveis (DST) entre as mulheres atendidas nos Ambulatórios de Moléstias Infecciosas em Ginecologia e Obstetrícia do Hospital das Clínicas da Faculdade de Medicina de Ribeirão Preto da Universidade de São Paulo, 2007-2012.

\begin{tabular}{lrrrrr}
\hline & \multicolumn{2}{c}{ Sim } & & \multicolumn{2}{c}{ Não } \\
\cline { 2 - 3 } \cline { 6 - 7 } DST & $\mathbf{n}$ & \% & & n & \% \\
\hline Gonorreia & 7 & 0,47 & & 1463 & 99,53 \\
Hepatite B & 12 & 0,82 & & 1458 & 99,18 \\
Hepatite C & 29 & 1,97 & & 1441 & 98,03 \\
Herpes & 26 & 1,77 & & 1444 & 98,23 \\
HIV & 530 & 36,10 & & 940 & 63,90 \\
HPV & 312 & 21,20 & & 1158 & 78,80 \\
Sífilis & 47 & 3,20 & & 1423 & 96,80 \\
Tricomonas & 9 & 0,61 & & 1461 & 99,39 \\
\hline
\end{tabular}

O tabagismo foi informado por 31,5\% (462/1468) das mulheres, enquanto o alcoolismo e o uso de drogas ilícitas por respectivamente $14,7 \%$ e $16,0 \%$ delas.

O motivo da consulta, na ocasião do preenchimento do questionário aplicado às mulheres, era: 480 estavam gestantes (32,6\%); 530 eram infectadas pelo HIV (36,0\%); 446 apresentavam condiloma vulvar, perianal, vaginal, cervical ou em duas ou mais destas regiões $(30,3 \%) ; 452$ apresentavam neoplasia intraepitelial cervical grau I, II ou III (30,7\%); 222 apresentavam citologia alterada $(15,1 \%)$ e 19 haviam sido vítimas de violência sexual $(1,3 \%)$. Manifestações devido à infecção pelo HPV estavam presentes em 1008 mulheres $(68,6 \%)$. Entre as mulheres com NIC, $159(35,2 \%)$ apresentavam lesão de alto grau (NIC III) e idade entre 15 e 63 anos.

Dentre as mulheres incluídas no estudo, 750 $(51,0 \%)$ afirmaram já terem ouvido falar sobre HPV (tabela 3). Destas, 65,3\% sabiam da relação do patógeno com o câncer de colo uterino e 81,5\% afirmaram saber que o HPV era transmitido sexualmente. A média de idade dessas mulheres era de 31,3 anos, $59,7 \%$ eram casadas/amasiadas, escolaridade acima de 12 anos foi relatado por 13,0\% dessas mulheres, entre 5 e 8 anos por $37,8 \%$, entre 9 e 11 anos por $36,6 \%$. O motivo da consulta de $35,5 \%$ dessas mulheres foi infecção pelo HIV, 26,7\% estavam gestantes, $27,6 \%$ apresentavam neoplasia intraepitelial cervical grau I, II ou III, 14,9\% condiloma vulvar, perianal, vaginal, cervical ou em duas ou mais destas regiões. A resposta sobre o meio de obtenção das informações sobre o vírus estava preenchida em 719 casos. A maioria das mulheres (45\%) obteve conhecimento sobre o vírus por meio do médico (323/719); 165 delas ouviram na televisão; 100 por leitura de revista; 49 por meio de amiga e 24 devido à leitura de jornal. Dentre aquelas 323 que ouviram sobre o HPV por meio do médico, 236 apresentavam alguma manifestação pelo vírus; das 87 mulheres que não apresentavam manifestação, 61 haviam recebido anteriormente o diagnóstico de alguma outra DST. De 720 mulheres que não haviam ouvido falar sobre HPV, $51,7 \%$ apresentavam escolaridade entre 5 e 8 anos, $25,9 \%$ escolaridade entre 9 e 11, e 13,8\% entre 0 e 4 anos. Não houve diferença estatisticamente significante nas médias de idades entre o grupo que havia ouvido falar de HPV e o que não (teste $t$ de Student, $\mathrm{p}=0,0548$ ). Houve diferença estatisticamente significante na proporção de mulheres que haviam ouvido sobre HPV daquelas que não haviam ouvido quanto à manifestação de HPV ( $\mathrm{p}=0,0006)$, com maior proporção de manifestação de HPV naquelas que disseram ter ouvido sobre o vírus.

Tabela 3. Conhecimento sobre HPV entre as mulheres atendidas nos Ambulatórios de Moléstias Infecciosas em Ginecologia e Obstetrícia do Hospital das Clínicas da Faculdade de Medicina de Ribeirão Preto da Universidade de São Paulo, 2007-2012.

\begin{tabular}{lccccc}
\hline \multirow{2}{*}{ Variáveis } & \multicolumn{2}{c}{ Sim } & & \multicolumn{2}{c}{ Não } \\
\cline { 2 - 3 } \cline { 6 - 7 } Já ouviu falar de HPV? & n & \% & & n \\
Sabia da relação de HPV com o câncer de colo? & 490 & 51,0 & & 720 & 49,0 \\
Sabia da transmissão sexual do HPV? & 611 & 31,3 & & 980 & 66,7 \\
\hline
\end{tabular}




\section{Discussão}

Os resultados mostraram que a maioria das mulheres participantes do estudo estava na fase reprodutiva, apresentava baixa escolaridade, parceiro fixo e teve iniciação sexual em idade precoce. Os fatores baixa escolaridade e início precoce da atividade sexual são considerados de risco para aquisição de HPV/DST. ${ }^{7}$ Outros fatores de risco associados à infecção e persistência do HPV são: multiplicidade de parceiros sexuais, tabagismo ${ }^{10,11,12}$, coinfecção por agentes infecciosos como herpes simples tipo $2^{10}$, Chlamydia trachomatis e HIV, multiparidade ${ }^{10,13} \mathrm{e}$ comportamento sexual do parceiro. ${ }^{14}$

Já ter ouvido falar de HPV foi afirmado por $51,0 \%$ das mulheres, todavia, apenas $41,6 \%$ do total de mulheres sabiam da transmissão sexual do patógeno e apenas $33,3 \%$ da relação do vírus com o desenvolvimento de câncer do colo uterino. Com esses resultados, é possível inferir que há deficiências no conhecimento relacionado ao assunto. Neste estudo, ter ouvido falar sobre HPV esteve significativamente associado ao diagnóstico prévio ou atual de alguma manifestação pelo vírus. Este achado pode ser explicado pelo fato de que as mulheres diagnosticadas com infecção pelo HPV na rede básica de saúde são encaminhadas ao AMIGO ou ao AMIG do HC-FMRPUSP para diagnóstico e tratamento apropriado. Ao serem referenciadas, provavelmente as pacientes recebem do médico orientações e explicações sobre seu diagnóstico e com isso ouvem sobre o HPV. Segundo, $45 \%$ das pacientes apontaram o profissional médico como a fonte das informações, reforçando a figura do médico como educador em saúde. Esta função do profissional, apesar de não ser considerada sua principal função, é uma das mais importantes.

A falta de conhecimentos adequados sobre o HPV é capaz de produzir informações erradas que podem dificultar ações de prevenção de doenças e promoção de saúde, além de causar medo e prejudicar o bem-estar das portadoras do HPV devido a crenças, mitos e tabus. ${ }^{15}$ Avaliando uma população de mulheres que fazem sexo com mulheres, Bertolin e colaboradores $(2010)^{8}$ constataram que $68 \%$ não sabiam o que significava HPV e $58 \%$ acreditavam que o uso de preservativo protege totalmente contra esta infecção viral, com isso evidenciaram vulnerabilidade para infecção pelo HPV devido a fatores de risco aos quais se expõem. Análise prévia da população de gestantes portadoras do papilomavírus humano realizada no AMIGO HC-FMRP-USP, observou que a faixa etária esteve entre 17 e 40 anos, 44,4\% das gestantes relataram escolaridade compatível com $1^{\circ}$ grau incompleto e $57,4 \%$ das mulheres atendidas no pré-natal nunca tinham ouvido falar do HPV. ${ }^{16}$ Estudo realizado com mulheres entre 40 e 60 anos de idade sem manifestações por HPV constatou deficiência no conhecimento dessas mulheres sobre o HPV. ${ }^{17}$ Estes estudos demonstram que apesar de tratar-se de uma das DST de etiologia viral mais frequente em todo o mundo, o conhecimento da população sobre a infecção pelo HPV ainda é deficiente e para Silva e Vargens $(2009)^{18}$ a falta de informação é um dos fatores de vulnerabilidade das mulheres para aquisição de DST.

As mulheres consideram as mulheres no geral vulneráveis a contraírem DST/HIV, porém não consideram elas mesmas em risco, caracterizando a ideia de vulnerabilidade e de doença do outro. Por não se considerarem vulneráveis acabam não utilizando preservativos por confiarem no parceiro. ${ }^{18} \mathrm{Se}$ as mulheres não se sentem vulneráveis a uma doença, elas costumam não aceitar medidas de prevenção a essa doença. Em alguns casos, a percepção de vulnerabilidade à doença associa-se com a diminuição de comportamentos de risco. Em outros casos, apesar da consciência de vulnerabilidade de si mesmas, o comportamento de risco se mantém. ${ }^{7}$

Estudos também mostram carência de conhecimento das mulheres sobre o câncer de colo útero, em especial sobre os fatores de risco e prevenção $0^{9,11,19}$ e sobre prevenção das DST. ${ }^{19}$ Proporções mais altas de conhecimento e atitudes adequadas parecem estar associadas com idade maior ou igual a 35 anos e escolaridade maior ou igual a nove anos. ${ }^{20}$ Thum et al. $(2008)^{11}$ observaram a televisão como principal fonte de informações.

$\mathrm{O}$ acesso às informações sobre a infecção pelo HPV assim como a educação da população e dos profissionais de saúde sobre esta DST, associados a medidas comprovadamente eficazes como a vacinação para HPV são de fundamental importância para o controle da transmissão viral e prevenção do câncer de colo uterino. De acordo com Czeresnia (1999) ${ }^{21}$, educação em saúde não é resolvida simplesmente com informação e capacitação técnica, mas deve ser estruturada por circulação de conhecimento científico e recomendações normativas para mudança de hábitos. Educação em saúde busca provocar mudanças no comportamento individual e é entendida como combinações de experiências de aprendizagem ordenada- 
mente projetadas com vistas a predispor, permitir e reforçar atitudes comportamentais adotadas com total compreensão e aceitação dos objetivos das ações desenvolvidas e aconselhadas que conduzam à própria saúde. ${ }^{22}$ Para isso são indispensáveis atividades de educação em saúde ou aconselhamento, com objetivo de fazer com que a população alvo compreenda e assimile os fatores de risco associados, principalmente os relacionados ao comportamento sexual. ${ }^{23} \mathrm{Po}-$ rém, neste estudo, 59,3\% das mulheres incluídas apresentaram baixa escolaridade (até 8 anos), fator que pode dificultar a realização de medidas preventivas e de promoção de saúde da mulher de acordo com Moura e colaboradores (2010). ${ }^{19}$
Com isso, pode-se concluir que a população de mulheres deste estudo apresenta deficiência no conhecimento sobre o HPV, apontando para a necessidade de ações educativas voltadas para a saúde com a finalidade de informar a população de maneira clara e abrangente promovendo maior autocuidado e menores taxas de morbidade. Para a implementação destas ações o envolvimento dos profissionais de saúde como os médicos, apontados neste estudo por $45 \%$ das mulheres como a fonte de informação, assim como a utilização da televisão como veículo de comunicação, apontada por $23 \%$ das mulheres, são de fundamental importância para promover o conhecimento e consequentemente a saúde da mulher.

\begin{abstract}
Study design: cross-sectional Objective: the current study aimed to assess the knowledge of women seen in two outpatient clinic of gynecology and obstetrics about the Human Papilloma Virus (HPV). Methodology: using secondary data from an electronic database that information was collected through a questionnaire during the period from 2007 to 2012. Results: were included 1470 women with average age of 30.7 years. Among women, $51.0 \%$ had heard about HPV, but only $41.6 \%$ knew of its sexual transmission and $33.3 \%$ knew about the relation of the pathogen with cervical cancer. Conclusion: data allowed to conclude that knowledge about HPV is poor, requiring improvement of educational interventions for the prevention of HPV infection and its worsening.
\end{abstract}

Keywords: Papillomavirus Infections. Knowledge. Women's Health.

\section{Referências Bibliográficas}

1. Koutsky L. Epidemiology of genital human papillomavirus infection. Am J Med 1997; 102(5A):3-8.

2. Schiffman $\mathrm{MH}$. Recent Progress in defining the epidemiology of human papillomavirus infection and cervical neoplasia. $J$ Natl Cancer Inst. 1992; 84: 394-8.

3. Kjaer SK, Svare EI, Worm AM, Walboomers JM, Meijer CJ, van den Brule AJ. Human papillomavirus infection in Danish female sex workers. Decreasing prevalence with age despite continuously high sexual activity. Sex Transm Dis. 2000; 27:438-45

4. Bruni L, Diaz M, Castellsagué X, Ferrer E, Bosch FX, de Sanjosé S. Cervical human papillomavirus prevalence in 5 continents: meta-analysis of 1 million women with normal cytological findings. J Infect Dis 2010; 202: 1789-99.

5. Myers ER, McCrory DC, Nanda K, Bastian L, Matchar DB. Mathematical model for the natural history of human papillomavirus infection and cervical carcinogenesis. Am J Epidemiol. 2000;151:1158-71.

6. Smith EM, Johnson SR, Ritchie JM, Feddersen D, Wang D, Turek LP, et ali. Persistent HPV infection in postmenopausal age women. Int J Gynaecol Obstet. 2004; 87:131-7.

7. Silveira MF, Béria JU, Horta BL, Tomasi E. Autopercepção de vulnerabilidade às doenças sexualmente transmissíveis e aids em mulheres. Rev Saúde Pública. 2002; 36: 670-7.
8. Bertolin DC, Ribeiro RCHM, Cesarino CB, Silva DC, Prado DO Parro ES. Conhecimento de mulheres que fazem sexo com mulheres sobre o papilomavírus humano. Cogitare Enferm. 2010; 15: 730-5.

9. Pelloso SM, Carvalho MDB, Higarashi IH. Conhecimento das mulheres sobre o câncer cérvico-uterino. Acta Sci, Health Sci 2004; 26: 319-24.

10. Bosch FX, Sanjosé S. Chapter 1: Human Papillomavirus and Cervical Cancer-Burden and Assessment of Causality. J Natl Cancer Inst Monogr. 2003; 31:3-13.

11. Thum M, Heck RM, Soares MC, Deprá AS. Câncer de colo uterino: percepção das mulheres sobre prevenção. Ciênc Cuid Saúde. 2008; 7: 509-16.

12. Instituto Nacional de Câncer [homepage na Internet]. Estimativa 2012: Incidência de Câncer no Brasil. Ministério da Saúde. Brasília: 2012. [acesso em 16 set 2012]. Disponível em: http://www2.inca.gov.br/wps/wcm/connect/inca/portal/ home

13. Instituto Nacional de Câncer [homepage na Internet]. Estimativa 2010: Incidência de Câncer no Brasil. Ministério da Saúde. Brasília: 2010. [acesso em 20 jul 2011]. Disponível em: http://www2.inca.gov.br/wps/wcm/connect/inca/portal/ home

14. Bosch FX, Lorincz A, Muñoz N, Meijer CJLM, Shah KV. The causal relation between human papillomavirus and cervical cancer. J Clin Pathol. 2002; 55: 244-65. 
15. Sousa LB, Pinheiro AKB, Barroso MGT. Ser mulher portadora do HPV: uma abordagem cultural. Rev Esc Enferm USP. 2008; 42: $737-43$.

16. Tanaka EZ, Melli PPS, Bonaldo CD, Quintana SM. Avaliação do conhecimento de alguns aspectos da infecção pelo papilomavirus (HPV) entre gestantes portadoras do HPV. Ensaios e Ciência: Ciências Biológicas, Agrárias e da Saúde 2010; 14: 9-19.

17. Silveira CF, Melo MM, Rodrigues LR, Parreira BDM. Conhecimento de mulheres de 40 a 60 anos sobre o Papillomavirus Humano. Rev RENE. 2011; 12: 309-15.

18. Silva $\mathrm{CM}$, Vargens OMC. A percepção de mulheres quanto à vulnerabilidade feminina para contrair DST/HIV. Rev Esc Enferm USP. 2009; 43: 401-6.

19. Moura ADA, Silva SMG, Farias LM, Feitoza AR. Conhecimento e motivações das mulheres acerca do exame de Papanicolaou: subsídios para a prática da enfermagem. Rev RENE. 2010; 11: 94-104.
20. Vasconcelos CTM, Pinheiro AKB, Castelo ARP, Costa LQ, Oliveira RG. Conhecimento, atitude e prática relacionada ao exame colpocitológico entre usuárias de uma unidade básica de saúde. Rev Latinoam Enferm. 2011; 19: 97-105.

21. Czeresnia D. The concept of health and the difference between prevention and promotion. Cad Saúde Pública. 1999; 15: 701-9.

22. Candeias NMF. Conceitos de educação e de promoção em saúde: mudanças individuais e mudanças organizacionais. Rev Saúde Pública. 1997, 31: 209-13.

23. Queiroz DT, Pessoa SMF, Sousa RA. Infecção pelo Papiloma Vírus Humano (HPV): incertezas e desafios. Acta Paul Enferm. 2005; 18:190-6. 